MCTP-02-72

\title{
Revisiting Top-Bottom-Tau Yukawa Unification in Supersymmetric Grand Unified Theories
}

\author{
Kazuhiro Tobe and James D. Wells \\ MCTP, Department of Physics, University of Michigan, Ann Arbor, MI 48109, \\ Department of Physics, University of California, Davis, CA 95616
}

\begin{abstract}
Third family Yukawa unification, as suggested by minimal $S O(10)$ unification, is revisited in light of recent experimental measurements and theoretical progress. We characterize unification in a semi-model-independent fashion, and conclude that finite $b$ quark mass corrections from superpartners must be nonzero, but much smaller than naively would be expected. We show that a solution that does not require cancellations of dangerously large $\tan \beta$ effects in observables implies that scalar superpartner masses should be substantially heavier than the $Z$ scale, and perhaps inaccessible to all currently approved colliders. On the other hand, gauginos must be significantly lighter than the scalars. We demonstrate that a spectrum of anomaly-mediated gaugino masses and heavy scalars works well as a theory compatible with third family Yukawa unification and dark matter observations.
\end{abstract}

hep-ph/0301015

January 2003 


\section{Introduction}

The unification of the gauge couplings in supersymmetric (SUSY) theories leads one to entertain the possibility that the three gauge groups of the Standard Model unify into a simple grand unified group. Computations within this framework have shown that the lowenergy gauge couplings are not only compatible with supersymmetric unification, but are predicted accurately to within expected slop of a sensible high-scale theory.

Any unified theory of the leptons and quarks gives a symmetry reason for their masses once the unified theory is broken and parameters are renormalized to the low scale where we take measurements. The pressing issue then is to use what we know to posit more complete forms of the high-scale theory in order to make more predictions and identify more tests for consistency.

We have mild clues to the form of the high-scale theory. First, the unification of gauge couplings works provided the hypercharge of each state is normalized as though it came from $S U(5)$ breaking. This makes any simple group which has an $S U(5)$ subgroup up for primary consideration, such as $S U(5), S O(10), E_{6}$, etc. Second, the existence of neutrino masses gives preference to theories that naturally incorporate the left and right neutrinos into simple representations. For this reason, we wish to focus on $S O(10)$, which is the smallest rank symmetry group that pays full respect to these two clues.

The simplest models of $S O(10)$ have complete unification of the third family Yukawa couplings at the high scale. This requirement implies the well-known criterion that $\tan \beta$ should be large (about 50) [1].

When $\tan \beta$ is large, the renormalization group evolution of the theory parameters (gauge couplings and Yukawa couplings) can only be done accurately by two-loop numerical computations. The numerics are somewhat messy, and so the hope was originally that the issue of Yukawa coupling unification could be elucidated by a compensatingly easy form of supersymmetry with few parameters. For example, one would have like to have seen how it all works for "minimal supergravity", where all the gauginos have the same mass at the high scale and all scalars have the same mass at the high scale. Unfortunately, this simple model fails to simultaneously allow Yukawa unification and electroweak symmetry breaking [2].

The lack of simple analytics to analyze Yukawa unification and the failure of a simple model to exemplify it has created a little confusion (for us, at least) on whether third family Yukawa unification is a reasonable expectation. The question has been studied by several groups in the past (for very recent papers, see Refs. [5, 6, 7) ); however, we are revisiting it because experimental input data on the strong coupling, the top mass, and the bottom mass has improved dramatically in the past few years, there are some discrepancies in the $b-\tau-t$ unification literature [3, 4, 5, 6, and the theoretical palette of supersymmetric models has grown. We also believe we have an effective way of showing in a semi-model-independent fashion what the requirements, and challenges, are for Yukawa unification, and we believe we have identified a general approach to the superpartner spectrum that is worth considering as 
a solution to the Yukawa unification problem. We illustrate the effectiveness of this approach within anomaly mediated supersymmetry breaking.

\section{Ultraviolet and infrared sensitivity of prediction}

When $\tan \beta$ is very high there is an extreme sensitivity of low-energy parameters on the high-scale prediction of Yukawa unification. This might sound vaguely disturbing at first, but in fact it is an opportunity we can exploit to narrow the low-scale options that work. In contrast, extreme ultraviolet sensitivity would be disastrous to progress since it would not matter what shuffling of parameters we do at the low scale, their effects would all be dwarfed by high-scale uncertainties.

We will demonstrate the infrared and ultraviolet sensitivity of the predictions below. We also hope to demonstrate a somewhat easier method to characterize the success or failure of third family Yukawa coupling unification. In the end, we hope we will have demonstrated two important conclusions to the reader: third family Yukawa unification is compatible with low-scale supersymmetry, but the low-scale finite corrections to the bottom quark must be smaller than naive expectations would have them be. This latter point translates into the requirements that large low-scale SUSY threshold corrections must either cancel to give a moderate or small-sized correction to the $b$-quark mass, or they must be greatly suppressed. When we discuss additional constraints on the low-scale spectrum from experiment, the suppression explanation will look more tenable in our view.

In order to understand how important the low-energy SUSY corrections are for Yukawa unification, we first show approximate expression of the GUT couplings, which depend on the low-energy SUSY corrections. The expressions for the gauge couplings are

$$
\begin{aligned}
g_{1}(M) \simeq & 0.73\left(1+3 \delta_{g_{1}}-0.007 \delta_{g_{2}}+0.02 \delta_{g_{3}}-0.02 \delta_{y_{t}}-0.005 \delta_{y_{b}}\right. \\
& \left.-0.002 \delta_{y_{\tau}}-0.007 \delta_{\tan \beta}+0.02 \log \frac{M}{M_{G_{0}}}+\delta_{g_{1}}^{G U T}+O\left(\delta^{2}\right)\right), \\
g_{2}(M) \simeq & 0.73\left(1-0.003 \delta_{g_{1}}+\delta_{g_{2}}+0.03 \delta_{g_{3}}-0.02 \delta_{y_{t}}-0.008 \delta_{y_{b}}\right. \\
& \left.-0.001 \delta_{y_{\tau}}-0.01 \delta_{\tan \beta}+0.004 \log \frac{M}{M_{G_{0}}}+\delta_{g_{2}}^{G U T}+O\left(\delta^{2}\right)\right), \\
g_{3}(M) \simeq \quad & 0.72\left(1-0.001 \delta_{g_{1}}-0.002 \delta_{g_{2}}+0.4 \delta_{g_{3}}-0.01 \delta_{y_{t}}-0.005 \delta_{y_{b}}\right. \\
& \left.-0.0002 \delta_{y_{\tau}}-0.005 \delta_{\tan \beta}-0.01 \log \frac{M}{M_{G_{0}}}+\delta_{g_{3}}^{G U T}+O\left(\delta^{2}\right)\right),
\end{aligned}
$$

where the scale $M$ should be close to the GUT scale $M_{G_{0}} \simeq 3 \times 10^{16} \mathrm{GeV}$, defined to be the location where $g_{1}\left(M_{G_{0}}\right)=g_{2}\left(M_{G_{0}}\right)$. Here $\delta^{G U T}$ is a threshold correction at GUT scale, and

$$
\delta_{g_{i}} \equiv \frac{\bar{g}_{i}^{M S S M}-\bar{g}_{i}^{S M}}{\bar{g}_{i}^{S M}},
$$


are deviations from the SM gauge couplings at $m_{Z}$ due to SUSY contributions. The uncertainties in the gauge coupling measurements at the $Z$ scale are also included in $\delta_{g_{i}}$ and must be taken into account when considering the predictions for unification. This is particularly true for the strong coupling constant $g_{3}$.

The parameterization of Yukawa threshold corrections is

$$
\delta_{y_{i}} \equiv \frac{\bar{y}_{i}^{M S S M}-\bar{y}_{i}^{S M} / \sin \beta_{0}}{\bar{y}_{i}^{S M} / \sin \beta_{0}}
$$

which keeps track of the deviations from the naive two Higgs doublet Yukawa couplings at $m_{Z}$ due to SUSY contributions $(\tan \beta=50)$. Similar to the gauge coupling parameters $\delta_{g_{i}}$, the Yukawa unification parameters take into account also the uncertainty of the fermion mass measurements. This is particularly important for $m_{b}$ and $m_{t}$, as their uncertainties are comparable to SUSY threshold corrections. Note that $\delta_{y_{i}}=\delta_{i}(i=b, t, \tau)$ as defined by Eqns. (33)-(35) in the Appendix. ${ }^{1}$ We have also defined

$$
\delta_{\tan \beta} \equiv \frac{\tan \beta-\tan \beta_{0}}{\tan \beta_{0}}
$$

where $\tan \beta_{0}=50$.

We can do a similar exercise for Yukawa couplings at a high-scale $M$ near $M_{G_{0}}$ to see qualitative features of Yukawa unification. Many numerical coefficients are highly sensitive to $\tan \beta$ and we have therefore expanded our results around $\tan \beta_{0}=50$ since we know that is near what $\tan \beta$ needs to be for third family unification to occur. The Yukawa couplings are then given by

$$
\begin{aligned}
y_{t}(M) \simeq & 0.63\left(1+0.9 \delta_{g_{1}}+3 \delta_{g_{2}}-3 \delta_{g_{3}}+7 \delta_{y_{t}}+0.7 \delta_{y_{b}}\right. \\
& \left.+0.02 \delta_{y_{\tau}}+0.7 \delta_{\tan \beta}-0.01 \log \frac{M}{M_{G_{0}}}+\delta_{t}^{G U T}+O\left(\delta^{2}\right)\right), \\
y_{b}(M) \simeq & 0.44\left(1+0.7 \delta_{g_{1}}+2 \delta_{g_{2}}-2 \delta_{g_{3}}+\delta_{y_{t}}+3 \delta_{y_{b}}+0.2 \delta_{y_{\tau}}\right. \\
& \left.+3 \delta_{\tan \beta}-0.02 \log \frac{M}{M_{G_{0}}}+\delta_{b}^{G U T}+O\left(\delta^{2}\right)\right) \\
y_{\tau}(M) \simeq & 0.52\left(1+0.1 \delta_{g_{1}}+\delta_{g_{2}}-0.6 \delta_{g_{3}}+0.2 \delta_{y_{t}}+\delta_{y_{b}}+2 \delta_{y_{\tau}}\right. \\
+ & \left.3 \delta_{\tan \beta}-0.005 \log \frac{M}{M_{G_{0}}}+\delta_{\tau}^{G U T}+O\left(\delta^{2}\right)\right)
\end{aligned}
$$

These expression should not be used in quantitative analysis because $O\left(\delta^{2}\right)$ corrections to Yukawa couplings are not negligible. But they do demonstrate interesting qualitative features. For gauge couplings, unification is not terribly sensitive to low energy SUSY corrections (i.e., the coefficients of $\delta_{y_{i}}$ and $\delta_{g_{i}}$ are small). On the other hand, Yukawa

\footnotetext{
${ }^{1}$ Our SUSY threshold corrections to Yukawa couplings contain logarithmic corrections from the wave function renormalization of the fermions as well as the finite corrections.
} 
couplings at the GUT scale are very sensitive to the low-energy SUSY corrections. An $O(1 \%)$ correction at low energies can generate close to a $O(10 \%)$ correction at the GUT scale. This extreme IR sensitivity is one source of the variance in conclusions in the literature [3, 4, 5, 6]. For example, course-grained scatter plot methods, which are so useful in other circumstances, lose some of their utility when IR sensitivity is so high. Furthermore, analyses that use only central values of measured fermion masses do not give a full picture of what range of supersymmetry parameter space enables third family Yukawa unification, since small deviations in low-scale parameters can mean so much to the high-scale theory viability.

\section{Semi-model-independent analysis of corrections}

We can make progress in understanding unification of the Yukawa couplings prior to positing a specific set of supersymmetric masses, and even prior to agreeing on a supersymmetry breaking framework. The technique we utilize is to consider the SUSY corrections $\delta_{t, b, \tau}$ as free parameters. We vary $\delta_{t, b, \tau}$ and $\tan \beta$ at $m_{Z}$, and search for $b-\tau-t$ Yukawa coupling unification at the GUT scale. For this analysis we define the GUT scale to be the scale where $g_{1}$ and $g_{2}$ unify. For the SUSY corrections to gauge couplings $\delta_{g_{i}}$, we use the formulas Eqns. (26) and (27) in the Appendix, assuming that all SUSY particle masses are $1 \mathrm{TeV}$. Later, we will also discuss the dependence of $\delta_{g_{3}}$.

Requiring exact Yukawa unification, we get the constraints among $\delta_{t, b, \tau}$ shown in Fig. 17. The contours are of $\delta_{b}$ values such that given a $\delta_{\tau}$ value ( $x$ axis) and a $\delta_{t}$ value ( $y$ axis) we get equality of the Yukawa couplings at the GUT scale. Fig. 1 lo reveals the $\tan \beta$ value needed to get unification for the given set of $\delta_{f}$ values. As expected, the allowed values of $\tan \beta$ are quite high and are consistent with previous analyses.

The size of corrections for $\delta_{t}$ and $\delta_{\tau}$ in typical weak scale supersymmetric theories with $\tan \beta \sim 50$ are roughly

$$
\begin{aligned}
\left|\delta_{t}\right| & \simeq\left|\frac{g_{3}^{2}}{6 \pi^{2}} \log \left(\frac{m_{Z}}{M_{\mathrm{SUSY}}}\right)\right| \lesssim 10 \% \\
\delta_{\tau} & \sim \frac{g_{2}^{2}}{32 \pi^{2}} \frac{M_{2} \mu \tan \beta}{M_{\mathrm{SUSY}}^{2}} \lesssim \mathrm{few} \%
\end{aligned}
$$

Therefore, one important point to notice in these graphs is the relatively small corrections allowed for $\delta_{b}$ compared to the expectation of $\left|\delta_{b}\right| \sim\left(g_{3}^{2} / 12 \pi^{2}\right) \tan \beta \sim 50 \%$ for high-tan $\beta$ supersymmetric theories with masses less than about a $\mathrm{TeV}$ and gauginos and sfermions roughly equal in mass.

The uncertainties and corrections in the strong coupling constant $\left(g_{3}\right)$ also play a role in the analysis, but do not change the qualitative picture. We expect the combination of weak-scale SUSY threshold corrections and the measurement uncertainty to be below about $\left|\delta_{g_{3}}\right| \lesssim 10 \%$. From Fig. 2 we see that the values of the $b$ quark corrections are still required to be small, $\delta_{b} \lesssim 10 \%$, and $\tan \beta$ is still near 50 . 


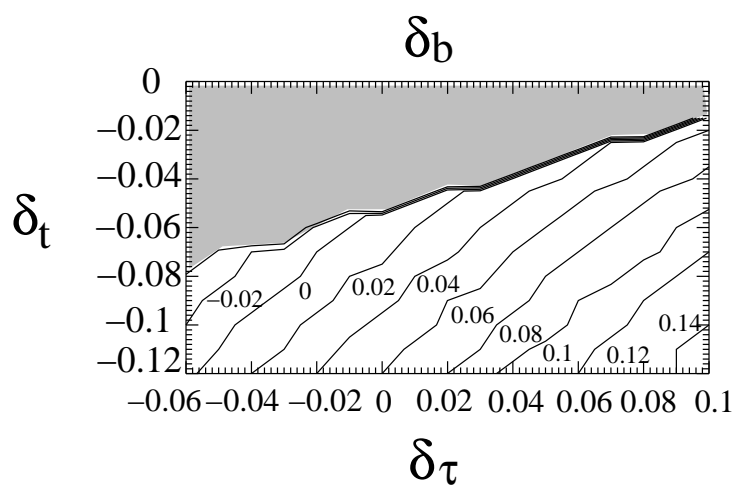

(a)

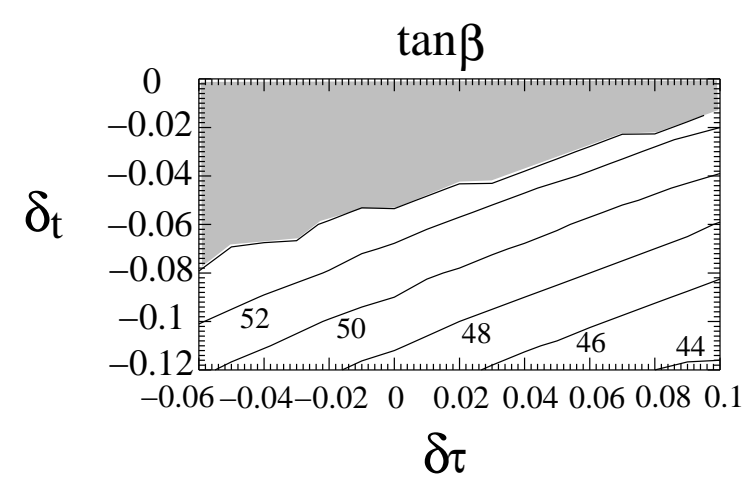

(b)

Figure 1: (a) SUSY threshold corrections $\delta_{b}$ to the bottom Yukawa coupling at $m_{Z}$, required for the Yukawa coupling unification, as a function of the SUSY threshold corrections to the top Yukawa coupling $\left(\delta_{t}\right)$ and tau Yukawa coupling $\left(\delta_{\tau}\right)$. (b) Contours of $\tan \beta$ needed for Yukawa unification in the parameter space of (a). The shaded region is where the needed value of $\tan \beta$ gets so high that the $b$ Yukawa coupling goes non-perturbative below the GUT scale.

Exact unification of the renormalized weak-scale Yukawa couplings at the high scale is not expected. The renormalized weak-scale Yukawa couplings will flow up to the high scale and be slightly mismatched, but the high-scale threshold corrections will shift them such that they unify. We therefore only need to require that the renormalized couplings flow within a reasonable neighborhood of each other, where reasonable neighborhood is defined to be size of the mismatch expected from the high-scale threshold corrections. In our above analysis, we have not included neutrino Yukawa coupling effects. If the tau neutrino Yukawa coupling is also unified with others at GUT scale, the right-handed neutrino mass scale $\left(M_{R}\right)$ should be around $10^{13}-10^{15} \mathrm{GeV}$ to explain atmospheric neutrino mass scale (assuming hierarchical neutrino masses). Therefore the right-handed neutrino scale is close to GUT scale. Here we take the neutrino Yukawa running effect as a GUT threshold effect. From one loop $\beta$-functions, the corrections are estimated to be at most a few $\%$ in the positive direction:

$$
\begin{aligned}
\delta_{t}^{G U T} & \simeq \delta_{\tau}^{G U T} \simeq \frac{y_{\nu}^{2}}{16 \pi^{2}} \log \frac{M_{G}}{M_{R}}, \\
\delta_{b}^{G U T} & \simeq 0 .
\end{aligned}
$$

It is hard to anticipate all high-scale threshold corrections, but it has been argued that Yukawa corrections at the high-scale are small, not more than a few percent [5]. To illustrate the effect this has on the low-scale theory predictions for $\tan \beta$ and the bottom quark mass corrections, we have chosen typical values of $\delta_{t}$ and $\delta_{\tau}$ weak-scale threshold corrections and plotted in Fig. [3] contours of $\tan \beta$ and $\delta_{b}$ for various sizes of high-scale threshold corrections.

The center vertical solid line in the figure corresponds to no high-scale threshold correc- 


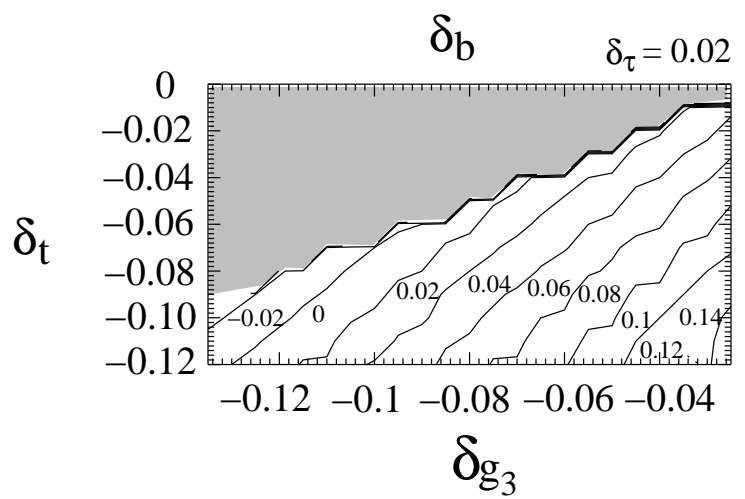

(a)

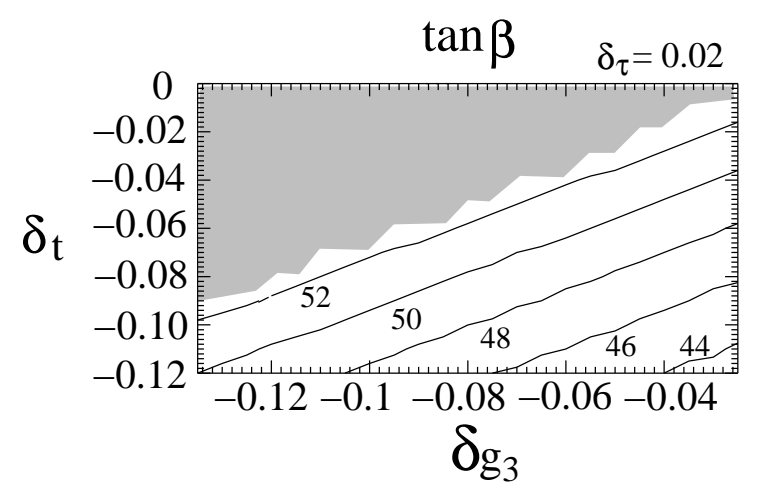

(b)

Figure 2: (a) SUSY threshold corrections $\delta_{b}$ at $m_{Z}$ required for Yukawa coupling unification, as a function of the top Yukawa SUSY threshold corrections $\left(\delta_{t}\right)$ and SUSY threshold corrections to the strong gauge coupling $\left(\delta_{g_{3}}\right)$. Here we fix $\delta_{\tau}=0.02$. (b) Contours of $\tan \beta$ to achieve Yukawa coupling unification in the parameter space of (a). The shaded region is where the needed value of $\tan \beta$ gets so high that the $b$ Yukawa coupling goes non-perturbative below the GUT scale.

tions to $\left[y_{b}\left(M_{G}\right)-y_{\tau}\left(M_{G}\right)\right] / y_{b}\left(M_{G}\right)$. The center horizontal solid line in the figure corresponds to no high-scale threshold corrections to $\left[y_{t}\left(M_{G}\right)-y_{\tau}\left(M_{G}\right)\right] / y_{t}\left(M_{G}\right)$. The various other lines are spaced at $5 \%$ threshold correction increments as one moves away from the center line. The solid parallelogram in the middle of the figure is the allowed region for $\tan \beta$ and $\delta_{b}$ given $5 \%$ threshold corrections at the high-scale. The bigger shaded region is for $10 \%$ threshold corrections. These reasonable, and perhaps even large, high-energy threshold corrections still do not change the basic conclusion that we would like to make here: $\delta_{b}$ corrections are required to be small.

Remember, in the construction of these graphs no assumptions have been made on the superpartner spectrum, so it will be up to us now to find out what spectrum can accommodate these corrections. More to the point: how can we get small $\delta_{b} \sim 5 \%$ with such a large $\tan \beta \sim 50$ ?

\section{Superpartner spectrum requirements}

We have made statements in the previous section that when $\tan \beta \sim 50$ and superpartner masses are below about $1 \mathrm{TeV}$ we expect $b$ quark mass corrections to be much higher than the $\delta_{b} \sim 5 \%$ required for third family unification. A quick way to see this is in the contributions to the finite corrections which scales as

$$
\delta_{b}^{\text {finite }} \simeq-\frac{g_{3}^{2}}{12 \pi^{2}} \frac{\mu M_{\tilde{g}} \tan \beta}{m_{\tilde{b}}^{2}}+\frac{y_{t}^{2}}{32 \pi^{2}} \frac{\mu A_{t} \tan \beta}{m_{\tilde{t}}^{2}}+\ldots
$$




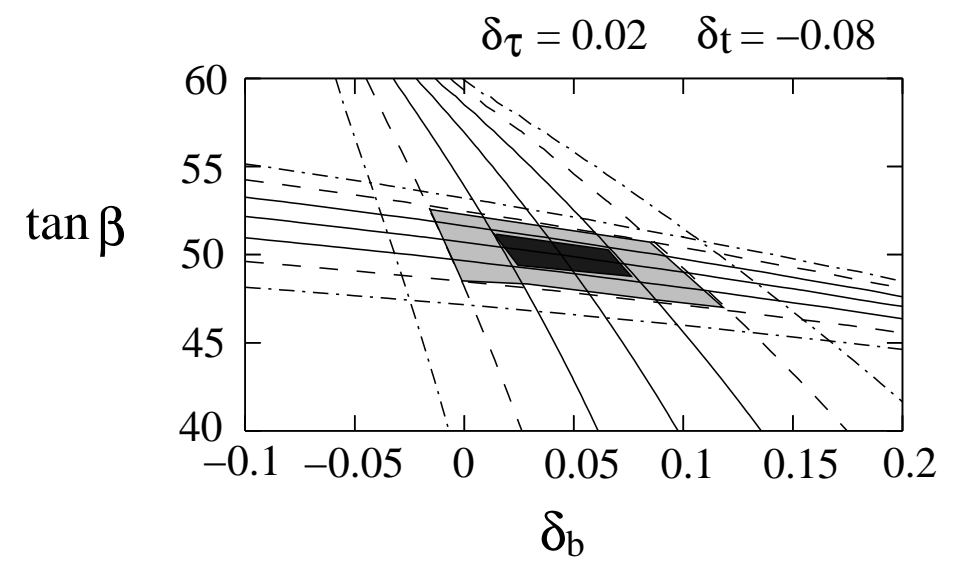

Figure 3: Contours of $\left[y_{b}\left(M_{G}\right)-y_{\tau}\left(M_{G}\right)\right] / y_{b}\left(M_{G}\right)$ (vertical lines) and $\left[y_{t}\left(M_{G}\right)-\right.$ $\left.y_{\tau}\left(M_{G}\right)\right] / y_{t}\left(M_{G}\right)$ (horizontal lines) as a function of $\delta_{b}$ and $\tan \beta$. We fix $\delta_{\tau}=0.02$ and $\delta_{t}=-0.08$. The dark-shaded region is the allowed region for Yukawa coupling unification, given $\pm 5 \%$ threshold corrections at the GUT scale. The bigger light-shaded region is for $\pm 10 \%$ threshold corrections.

If all superpartner masses are about the same, we see that each term contributes about $\delta_{b} \sim 50 \%$ [8, 9, 10, 11, 12] with the sign depending on the relative sign of $\mu M_{\tilde{g}}$ and $\mu A_{t}$.

To reduce the size of this correction to the $\delta_{b} \sim 5 \%$ level one needs to either cancel large individual terms in the formulas for the corrections, or suppress the corrections. In our view, the appeal of the first option is diminished, but not ruled out, when we contemplate the strong correlation between large SUSY contributions to $\delta_{b}$ and large SUSY contributions to $b \rightarrow s \gamma$.

We know from experiment that the decay rate $b \rightarrow s \gamma$ is consistent with the Standard Model [13, 14, and weak-scale supersymmetry (masses less than about a TeV) with large $\tan \beta$ supersymmetry generally is wildly in conflict with it. There are two ways out of this problem: the large individual SUSY corrections, including $t-H^{ \pm}, \tilde{t}-\tilde{\chi}^{ \pm}$and $\tilde{b}-\tilde{g}$ loop corrections, could conspire to cancel each other for a small total effect, or these same loop corrections could be so large that they are almost exactly twice the size of the SM amplitude, but opposite in sign so that the overall observable is not altered. This possibility is still allowed by the data, although future measurements of the FB asymmetry of $b \rightarrow s l^{+} l^{-}$ would distinguish the sign of the amplitude [14.

The terms in the $b \rightarrow s \gamma$ computation are very similar to the terms in the $\delta_{b}$ computation. They both involve loops of charginos and stops, and loops of gluinos and sbottoms (if supersymmetric CKM matrix is different than CKM matrix), but the coefficients and couplings are different. A cancellation of supersymmetric effects in one does not by any means imply a cancellation in the other. Therefore, it is perhaps reasonable for a low-scale supersymmetric model with $\tan \beta \sim 50$ to conspire to give small corrections to either $b \rightarrow s \gamma$ or to $\delta_{b}$, but 
we view it as unlikely that cancellations occur for both observables.

We are next led to the explanation that $\delta_{b}$ is small because all terms contributing to it are suppressed. It has been pointed out that an approximate $R$ symmetry or PQ symmetry can systematically suppress both $\delta_{b}$ and $b \rightarrow s \gamma$ [9]. Both computations go to zero as supersymmetric parameters carrying $R$-symmetry charge (gaugino masses and $A$ terms) go to zero, and both computations go to zero if terms carrying PQ charge (the $\mu$ term) go to zero.

The constraints from $b \rightarrow s \gamma$ are very severe at $\tan \beta \sim 50$. If all superpartner masses are less than $1 \mathrm{TeV}$ it is challenging to envision a superpartner spectrum capable of suppressing individual contributions enough to not require a rather large beneficial cancellation (conspiracy of amplitude signs) to occur in the finite $b$-quark to allow Yukawa unification. And in addition, we simultaneously need equivalently large contributions to the $b \rightarrow s \gamma$ amplitude to cancel or add with just the right strengths to recover the magnitude of the SM amplitude but with opposite sign.

The problem of making relatively light supersymmetry compatible with Yukawa coupling unification was taken up in the excellent study by Blažek, Dermíšek and Raby (BDR) [5]. ${ }^{2}$ The BDR approach is within a model of unified scalar mass $m_{16}$ at the high scale for all states in the 16 representation (squarks, sleptons, sneutrinos), split Higgs masses within the 10 representation, and unified gaugino masses $m_{1 / 2}$ at the GUT scale. They find that in order for third family Yukawa unification to occur the SUSY parameters must have the following properties:

1. $\tan \beta \sim 50$ for the third generation Yukawa couplings to approach unification.

2. $m_{1 / 2} \sim \mu \ll m_{16}$ to suppress, but not to zero, the gluino contributions to $\delta_{b}$ and $b \rightarrow s \gamma$.

3. Large $A_{0}$ such that the weak-scale $A_{t}$ is larger than $M_{\tilde{g}}$ and the positive chargino-stop contributions to $\delta_{b}$ cancels, and slightly overcomes, the large negative contributions due to gluino-sbottoms finite and logarithmic corrections. (Note, we are expressing this criteria in our sign convention for $b$-quark corrections which is opposite to BDR sign convention: $\left.\delta_{b} \propto-\Delta m_{b}^{\mathrm{BDR}}\right)$.

4. $\mu>0$ so that the large chargino-stop corrections to $b \rightarrow s \gamma$ can be opposite in sign to SM (and charged Higgs) contributions. This is necessary to be consistent with the large choice of $A_{0}$ term above, which when combined with the right sign of $\mu$ gives the chargino-stop loops a sufficiently large canceling contribution to change the sign of the $b \rightarrow s \gamma$ amplitude. This enables large $\tan \beta$ supersymmetry to be consistent with the $B(b \rightarrow s \gamma)$ measurements despite the SUSY contributions being much larger than the SM contributions.

\footnotetext{
${ }^{2}$ Similar findings for Yukawa unification were found in Ref. [6] using a D-term model approach and assuming larger possible GUT scale Yukawa corrections.
} 
The above criteria for third generation Yukawa unification may seem tortured to the uninitiated, but we can verify after much technical work in hopes of finding something "better" that the BDR solution is the right approach if supersymmetry is relatively light. It is remarkable how robustly unique of a general solution approach it turns out to be, and we concur with BDR on its interesting phenomenological predictions, such as $m_{h} \simeq 114 \pm 6 \mathrm{GeV}, m_{\tilde{t}_{1}} \ll m_{\tilde{b}_{1}}$, etc.

There is one disquieting feature of the BDR solution that one might call "finetuned cloaking of large $\tan \beta$ effects." It is unsettling to expect that there is a nice conspiracy of large effects coming out just right (and more or less SM-like) for $b$-quark finite corrections and $b \rightarrow s \gamma$ corrections. Large corrections would not be a cause for any concern whatsoever. However, large corrections that cancel to yield small $b$-quark corrections and add just the right amount to suppress or flip the sign of the $b \rightarrow s \gamma$ amplitude such that we would see no deviation from the SM may be too much to ask of a theory.

There are other, perhaps just as severe, challenges to the BDR solution. There may be another conspiracy involved in getting $g-2$ to work out properly. However, as BDR have pointed out, it is generally less severe than $b \rightarrow s \gamma$ considerations, and the required choice of $\mu>0$ at least goes in the preferred direction for $g-2$. A more difficult challenge would be to get acceptable dark matter solutions. When scalar masses are much larger than gaugino masses, the relic abundance of the stable lightest neutralino is typically much too large to be acceptable. Additional tunings of the spectrum and parameters would be required to make this work out properly. For example, just the right mixture of higgsino and bino components to the lightest neutralino would guarantee any amount of dark matter one wishes, but this requires a somewhat sensitive tuning between $m_{1 / 2}$ and $\mu$.

Annihilations of the lightest neutralino through a heavy Higgs pole is another viable option to suppress relic abundance in high $\tan \beta$ theories of this sort [6], but it requires the heavy Higgs states to be relatively light in contradistinction to other scalars in the theory. Nevertheless, the large $\tan \beta$ suppression of the pseudoscalar mass with respect to lagrangian parameters is encouraging for this scenario.

\section{The partially decoupled solution}

We would like to discuss another solution to third generation Yukawa unification that involves no finetuned cloaking of the large $\tan \beta$ effects. As the overall scale of supersymmetry breaking increases, the supersymmetric effects in $b \rightarrow s \gamma$ (and $g-2$ ) decouple to zero. However, the finite and logarithmic threshold corrections to the Yukawa couplings do not decouple.

Logarithmic corrections to $\delta_{b}$ and $\delta_{t}$ are negative and can be over $10 \%$ for squark masses above several $\mathrm{TeV}$. The logarithmic corrections to $\delta_{\tau}$ are negligible (less than $1 \%$ ). Large negative corrections to $\delta_{b}$ go in the wrong direction for Yukawa unification, and if they stood 
alone would invalidate the hypothesis of third generation Yukawa unification consistent with reasonable high-scale threshold corrections.

Once we realize that there are negative and large irrepressible logarithmic corrections to $\delta_{b}$ and $\delta_{t}$ it becomes clear that moderately valued finite corrections (about $10 \%$ to 20\%) to $\delta_{b}$ from gluino-squark loops must be present. This is a very reasonable request of the theory since the $\delta_{b}$ corrections depend only on ratios of masses and not on the overall scale of supersymmetry breaking: $\delta_{b} \propto-\mu M_{\tilde{g}} / m_{\tilde{b}}^{2}$. This non-decoupling property of the finite $b$ quark mass corrections is what allows us to suppress large $\tan \beta$ effects in potentially dangerous observables and yet get large enough finite $b$-quark mass corrections to obtain viable third family Yukawa unification.

The solution described above is transparently natural in every way except electroweak symmetry breaking might be finetuned because we need large scalar masses. For example, stop masses above a few $\mathrm{TeV}$ are needed for this solution to be realized in a way that naturally satisfies large $\tan \beta$ sensitive observables, such as $b \rightarrow s \gamma$.

We have identified anomaly mediated gaugino masses and $A$ terms [15, 16] as a theoretical scenario which may naturally realize this approach to third generation Yukawa unification. We utilize several features of this idea to our benefit. If no singlets are around to transmit supersymmetry breaking, the gaugino masses and $A$ terms (dimension 3 SUSY breaking terms) may primarily arise via anomaly mediation and are approximately one-loop suppressed compared to the gravitino mass, and hence the $A$ terms are naturally the size of the gaugino masses.

We also remind the readers that although the gaugino mass relations in anomaly mediation do not look like they have any connection to a GUT theory, they are in fact perfectly consistent with a GUT unified gaugino mass. The odd splitting of gaugino masses below the GUT scale comes from large gauge-mediated threshold corrections from integrating out heavy GUT states. The "magic" of anomaly mediation is that these corrections are precisely what is needed to recover the scale-invariant expressions for the gaugino masses at low energy.

On the other hand, the scalars can naturally get masses of the order the gravitino mass because $F^{\dagger} F / M_{\mathrm{Pl}}^{2}$ scalar mass-squareds do not require singlets. Scalar masses may be suppressed somewhat with respect to gravitino mass if the Kähler potential approaches the no-scale form [15, 16]. We therefore make the general observation that anomaly mediation is most naturally manifest by gauginos much lighter than scalars, although the precise ratio between them depends on a priori incalculable aspects of the theory (i.e., the Kähler potential).

To illustrate how Yukawa unification works in this scenario we normalize the anomaly mediated gaugino masses to $M_{2}=150 \mathrm{GeV}$. This is well above the experimental limits. (Recall that $\chi^{ \pm}-\chi_{1}^{0}$ near degeneracy makes experimental detection extremely challenging, and normal chargino limits do not apply [17.) This implies that $M_{1} \simeq 500 \mathrm{GeV}$ and $M_{\tilde{g}} \simeq$ $-1300 \mathrm{GeV}$. It also implies that the gravitino mass should be about $60 \mathrm{TeV}$. 


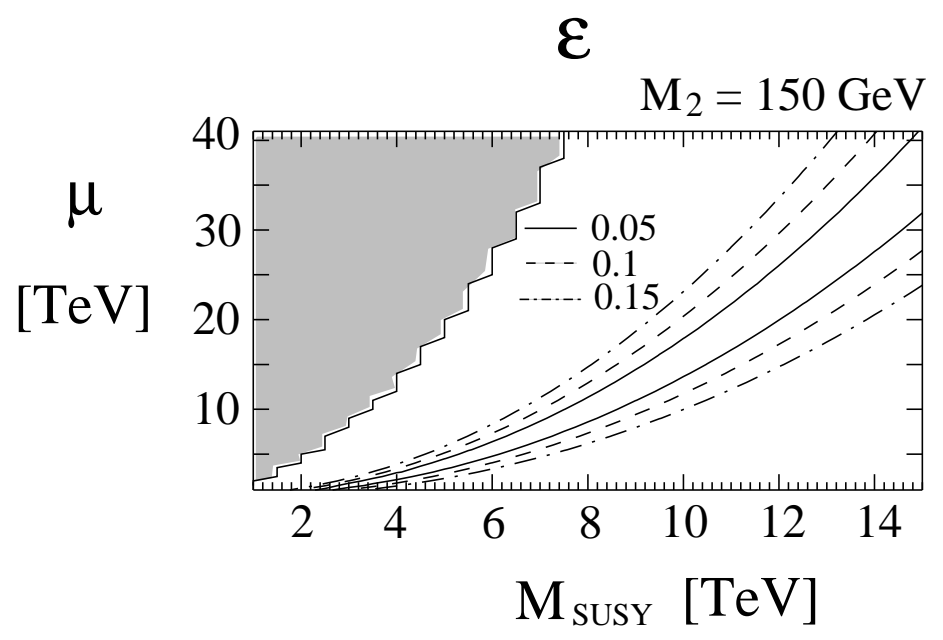

Figure 4: Contours of $\epsilon$ defined by Eq. (15) $) ~ \epsilon=0.05(0.1,0.15)$ corresponds to about $5 \%(10 \%, 15 \%)$ GUT threshold correction needed to achieve Yukawa coupling unification. GUT-scale Yukawa corrections are expected to be less than about 1\%. $M_{\text {SUSY }}$ is the lowenergy mass for all scalar superpartners. The gaugino and A-term masses are equal to their anomaly-mediated values normalized to $M_{2}=150 \mathrm{GeV}$.

In the next step we compute

$$
\epsilon=\sqrt{\left(\frac{y_{b}-y_{\tau}}{y_{b}}\right)^{2}+\left(\frac{y_{t}-y_{\tau}}{y_{t}}\right)^{2}+\left(\frac{y_{t}-y_{b}}{y_{t}}\right)^{2}} \quad \text { (computed at GUT scale) }
$$

as a function of the soft SUSY breaking sfermion masses (which we assume all to be degenerate $M_{\text {SUSY }}$ ) and $\mu$. Reasonable unification requires $\epsilon<0.05$. In Fig. 4 we plot contours of $\epsilon$ in the $M_{\mathrm{SUSY}}-\mu$ plane. We note that if we rescale $m_{\tilde{b}}\left(\simeq M_{\mathrm{SUSY}}\right.$ ) by a factor of $a$ then $\mu$ must be rescale by a factor of $a^{2}$ in order to keep the finite $\delta_{b} \propto-\mu M_{\tilde{g}} / m_{\tilde{b}}^{2}$ constant for a given value of $M_{\tilde{g}}$. Therefore, since we expect $\mu$ to not be much heavier than $m_{\tilde{b}}$ we could gather from Fig. 4 that the squark and $\mu$-term masses should be less than about $10 \mathrm{TeV}$ in this case. Combining that with our requirement that large $\tan \beta$ effects mostly decouple in $b \rightarrow s \gamma$ means we expect

$$
\left|M_{\tilde{g}}\right| \simeq 1300 \mathrm{GeV} \longrightarrow m_{\tilde{b}} \gtrsim \text { few TeV }
$$

In our case, the $A$ term value is quite small since it scales with the gaugino masses in anomaly mediation. Furthermore, we have assumed minimal flavor violation. Relaxing that requirement would tighten up the $b \rightarrow s \gamma$ very significantly, and push the required $b$-squark masses beyond $5 \mathrm{TeV}$ or more.

In general the expected ratio of $m_{\tilde{b}} / M_{\tilde{g}}$ needs to be between about 5 and 15 for Yukawa unification. For scalar masses right at the gravitino mass, this ratio would be about 45, so 
we need the scalar mass to be at least a factor of 4 suppressed compared to the gravitino mass. Again, this presumably would come from a Kähler potential that suppresses the mass, although not nearly so absolutely, and perhaps not so unnaturally, as the no-scale Kähler potential.

As stated earlier, we have computed the value of $B(b \rightarrow s \gamma)$ in this framework by assuming the minimal flavor violation, and we have applied the above supersymmetry spectrum to the formula of Ref. [13. We demonstrate in Fig. 5] that indeed the large $\tan \beta$ effects are easily decoupled in the interesting range of scalar masses above a few $\mathrm{TeV}$. The figure plots contours of constant

$$
R=\frac{B(b \rightarrow s \gamma)}{B(b \rightarrow s \gamma)_{\mathrm{SM}}} .
$$

The data requires that this ratio be within $0.37<R<1.25$ [13]. Our parameter space easily satisfies this bound.

Furthermore, we have checked that $g-2$ is easily consistent with the parameter space. A conservative view of the $g-2$ experimental uncertainties and theoretical uncertainties implies that $a_{\mu}^{\text {susy }} / 10^{-10}$ should be between about -37 and 90 [18. The predictions for $g-2$ given in Fig. 5] are certainly well within that range. A less conservative interpretation of the experiment and theory implies [19] that

$$
a_{\mu}^{\text {susy }} / 10^{-10}=34 \pm 11 \quad\left(e^{+} e^{-} \text {based analysis }\right) .
$$

Therefore, there is a slight preference for positive values of $g-2$ which our approach can accommodate.

Given the above analysis on $b \rightarrow s \gamma$ and $g-2$ of the muon, we feel comfortable concluding that Yukawa unification is consistent with our solution to third generation Yukawa unification.

The drawback of our approach is that it increases the apparent finetuning of radiative electroweak symmetry breaking since all the scalars are at least several TeV. We have made a trade compared to the BDR solution: we have rid ourselves of the several finetuned cloakings of large $\tan \beta$ effects and traded it in for a larger finetuning for EWSB.

The anomaly-mediated case we presented here is also happily consistent with the lightest supersymmetric particle being the dark matter. In this case, the lightest supersymmetric particle is a neutral Wino particle. The relic abundance calculated from thermal equilibrium evolution and freezeout of Winos yields a much too low relic abundance to be of cosmological interest. This is of course less problematic than the overclosure problem which faces a typical spectrum of the BDR solution. Nevertheless, it would have been disheartening if the perfectly good prospect of LSP dark matter is no longer even viable. Fortunately this is not the case. Non-thermal production of the lightest neutralino via late-time gravitino [20] or moduli [21] decays works surprisingly well and is easily compatible with the right amount of cold dark matter needed to explain astrophysical observations. 


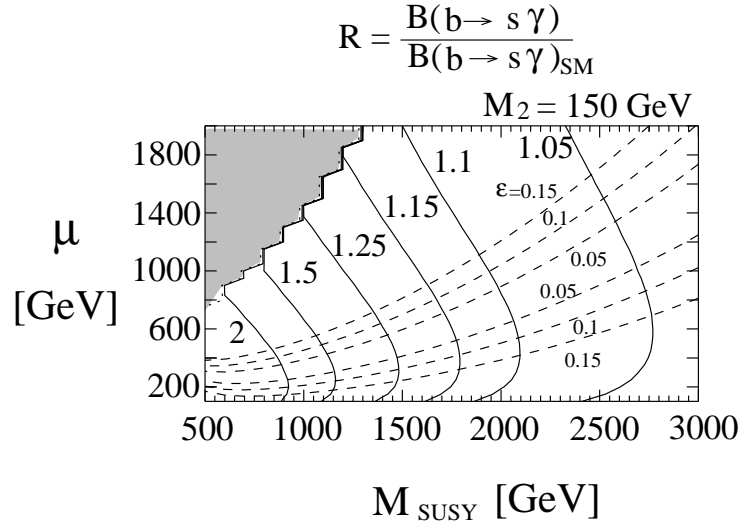

(a)

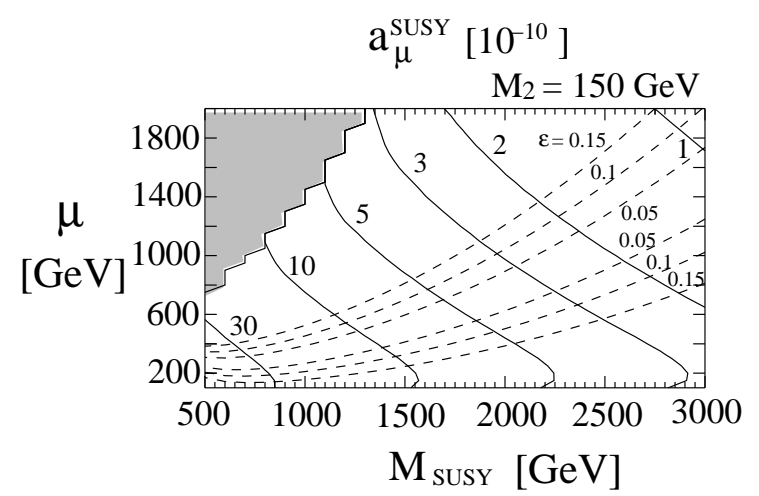

(b)

Figure 5: (a) Contours of $R=B(b \rightarrow s \gamma) / B(b \rightarrow s \gamma)_{\mathrm{SM}}$. (b) Contours of the SUSY contribution to muon $g-2\left(a_{\mu}^{\mathrm{SUSY}}\right)$. In Fig. (a) and (b), we also plot contours of $\epsilon(=$ 0.05, 0.1, 0.15) from Fig. 4. $M_{\mathrm{SUSY}}$ is the low-energy mass for all scalar superpartners. The gaugino and A-term masses are equal to their anomaly-mediated values normalized to $M_{2}=$ $150 \mathrm{GeV}$.

\section{Conclusion}

Supersymmetric grand unified theories are extremely attractive largely because gauge coupling unification works so well. The theory of low-energy supersymmetry is both compatible with gauge coupling unification and it is a predictive theory. Compatibility just means it is possible, and predictivity means it works surprisingly well - e.g., the low-scale value of $g_{3}$ is predicted to within a few percent even after taking into account the range of all reasonable high and low-scale threshold effects.

Similarly, low-scale supersymmetry is certainly compatible with third generation Yukawa unification, and, to a lesser extent, it is predictive. In this case, given that we now know all the SM fermion masses rather well, we can predict $\tan \beta$ and $\delta_{b}$ to interesting accuracies. These can then be traded in for knowledge/constraints on the superpartner spectrum.

Our analysis argues that a viable solution to Yukawa unification which has no finetuned cancellation of large $\tan \beta$ effects in observables requires very heavy scalar fields. We would say loosely that scalar masses are above several $\mathrm{TeV}$ if this idea is what nature realizes. We also require that either the $\mu$ term or the $R$ charged masses (gaugino masses and $A$ terms) must be light. A promising theory in this direction is anomaly mediated supersymmetry breaking, where the gaugino masses are predominantly anomaly mediated, and the scalar masses are somewhat suppressed compared to the gravitino mass. The compatibility with gauge coupling unification is assured, compatibility with all dangerous chirality-flipping observables is assured, and the LSP is retained as a viable dark matter candidate.

Finally, we have not found a superpartner spectrum that all would agree has no problems 
with finetuning. In the BDR approach, which we have verified as a viable solution, there is perhaps a finetuned cancellation of large $\tan \beta$ effects in observables. In our approach, there is perhaps a finetuned potential for electroweak symmetry breaking. We are painted in these two corners primarily because of recent experimental constraints (LEP, Tevatron, rare $B$ decays, precision electroweak, etc.). It would not be a totally indefensible position to say that experiment in the last decade has diminished the viability of third generation Yukawa unification. Our analysis could even be subpoened to support this view. Nevertheless, we believe that the extreme infrared sensitivity of Yukawa unification is a great opportunity to test the theory, and it should be of no surprise that such an IR sensitive concept would get severely squeezed shortly before it is redeemed or snuffed out by direct superpartner measurements.

\section{Acknowledgements}

K.T. thanks R. Dermíšek for useful discussions. K.T. and J.D.W. were supported in part by the U.S. Department of Energy.

\section{Appendix}

We describe the details of our analysis of Yukawa coupling unification. The first step is to compute gauge couplings and the third generation fermion masses in the $\overline{D R}$ scheme at the $Z$-boson mass scale $m_{Z}$, using the full standard model (SM).

For gauge couplings, we adopt experimental values [22] of the QED fine structure constant $\alpha^{-1}=137.06$, the hadronic contribution to the QED coupling at $m_{Z} \Delta \alpha_{\text {had }}^{(5)}\left(m_{Z}\right)=$ 0.02761, the leptonic effective electroweak mixing angle $\sin ^{2} \theta_{\text {eff }}^{\text {lept }}=0.23136$, and QCD coupling $\alpha_{s}^{(5)}\left(m_{Z}\right)=0.1172$ as input parameters, then we calculate the $\overline{M S}$ gauge couplings $\left(g_{i}^{S M} \overline{M S}\left(m_{Z}\right)\right)$ by using the formula in Refs. 23]. We convert them into the $\overline{D R}$ gauge couplings $\left(\bar{g}_{i}^{S M}\left(m_{Z}\right)\right)$ using the relation between $\overline{M S}$ and $\overline{D R}$ gauge couplings:

$$
\frac{4 \pi}{\bar{g}_{i}^{S M}\left(m_{Z}\right)^{2}}=\frac{4 \pi}{g_{i}^{S M} \overline{M S}\left(m_{Z}\right)^{2}}-\frac{C_{i}}{12 \pi},
$$

where $C_{1}=0, C_{2}=2$, and $C_{3}=3$.

For the bottom quark mass, we adopt the $\overline{M S}$ bottom quark mass $m_{b}^{\overline{M S}}$ at $m_{b}^{\overline{M S}}$ as an input parameter for our numerical analysis [24]:

$$
m_{b}^{\overline{M S}}\left(m_{b}^{\overline{M S}}\right)=4.26 \pm 0.30 \mathrm{GeV} .
$$

Using two-loop (and $\mathcal{O}\left(\alpha_{s}^{3}\right)$ for the QCD coupling) renormalization group (RG) analysis in the effective QCD and QED theory, we calculate the $\overline{M S}$ bottom quark mass at $m_{Z}$. At 
$m_{Z}$ we match the $\overline{M S}$ mass in the effective QCD and QED theory into one in the SM $\left(m_{b}^{S M} \overline{M S}\left(m_{Z}\right)\right)$ including electroweak contribution to the bottom quark. We then convert it into the $\overline{D R}$ bottom quark mass $\left(\bar{m}_{b}^{S M}\left(m_{Z}\right)\right)$ :

$$
\begin{aligned}
\bar{m}_{b}^{S M}\left(m_{Z}\right) & =m_{b}^{S M} \overline{M S}\left(m_{Z}\right)\left(1-\frac{\alpha_{s}\left(m_{Z}\right)}{3 \pi}-\frac{29 \alpha_{3}^{2}\left(m_{Z}\right)}{27 \pi^{2}}\right) \\
& =2.89 \pm 0.24 \mathrm{GeV}
\end{aligned}
$$

for $\alpha_{s}\left(m_{Z}\right)=0.1172$.

For the tau lepton mass, we adopt the pole mass $\left(m_{\tau}\right)$ as an input [24]:

$$
m_{\tau}=1776.99_{-0.26}^{+0.29} \mathrm{MeV}
$$

Then we calculate the $\overline{M S}$ tau mass at $m_{\tau}$, and we extrapolate it to one at $m_{Z}$ using two-loop $\mathrm{RG}$ analysis in the effective QED theory. At $m_{Z}$, we match it into one in the SM correcting for the electroweak contributions. Then we get the $\overline{D R}$ tau lepton mass at $m_{Z}$ :

$$
\bar{m}_{\tau}^{S M}\left(m_{Z}\right)=1748.77_{-0.26}^{+0.29} \mathrm{MeV} .
$$

For top quark mass, we use the pole mass $\left(m_{t}\right)$ as an input parameter:

$$
m_{t}=174.3 \pm 5.1 \mathrm{GeV} .
$$

Using a relation between the pole mass and $\overline{D R}$ mass in the SM at $m_{Z}$ scale, we compute the $\overline{D R}$ mass at $m_{Z}$ :

$$
\bar{m}_{t}^{S M}\left(m_{Z}\right)=172.3 \pm 5.3 \mathrm{GeV}
$$

for $\alpha_{s}\left(m_{Z}\right)=0.1172$.

In the next step, we compute $\overline{D R}$ couplings in the MSSM at $m_{Z}$. For gauge couplings, we include logarithmic SUSY threshold corrections ${ }^{3}$. The relation between the SM and MSSM $\overline{D R}$ gauge couplings is

$$
\frac{4 \pi^{2}}{\bar{g}_{i}^{M S S M}\left(m_{Z}\right)^{2}}=\frac{4 \pi^{2}}{\bar{g}_{i}^{S M}\left(m_{Z}\right)^{2}}+\Delta_{i}^{S U S Y}
$$

Here $\Delta_{i}^{S U S Y}$ is the SUSY logarithmic corrections:

$$
\begin{aligned}
\Delta_{3}^{S U S Y} & =\log \frac{m_{\tilde{g}}}{m_{Z}}+\frac{N_{g}}{3} \log \frac{m_{\tilde{q}}}{m_{Z}} \\
\Delta_{2}^{S U S Y} & =\frac{2}{3} \log \frac{m_{\tilde{W}}}{m_{Z}}+\frac{N_{g}}{4} \log \frac{m_{\tilde{q_{L}}}}{m_{Z}}+\frac{N_{g}}{12} \log \frac{m_{\tilde{l}_{L}}}{m_{Z}}
\end{aligned}
$$

\footnotetext{
${ }^{3}$ The SUSY finite corrections will be small if SUSY particle masses are more than a factor of a few larger than $m_{Z}$.
} 


$$
\begin{aligned}
& +\frac{1}{3} \log \frac{m_{\tilde{H}}}{m_{Z}}+\frac{1}{12} \log \frac{m_{H}}{m_{Z}}, \\
\Delta_{1}^{S U S Y}= & \frac{N_{g}}{60} \log \frac{m_{\tilde{q}_{L}}}{m_{Z}}+\frac{2 N_{g}}{15} \log \frac{m_{\tilde{u}_{R}}}{m_{Z}}+\frac{N_{g}}{30} \log \frac{m_{\tilde{d}_{R}}}{m_{Z}} \\
& +\frac{N_{g}}{20} \log \frac{m_{\tilde{l}_{L}}}{m_{Z}}+\frac{N_{g}}{10} \log \frac{m_{\tilde{e}_{R}}}{m_{Z}}+\frac{1}{5} \log \frac{m_{\tilde{H}}}{m_{Z}}+\frac{1}{20} \log \frac{m_{H}}{m_{Z}}
\end{aligned}
$$

In order to calculate the $\overline{D R}$ Yukawa couplings, we need the $\overline{D R}$ vacuum expectation value (vev) in the MSSM. In this paper, we define the $\overline{D R}$ vev $(\bar{v}(\mu))$ from the $\overline{D R}$ Z-boson mass:

$$
\bar{m}_{Z}^{2}(\mu)=\frac{\bar{g}^{\prime 2}(\mu)+\bar{g}_{2}^{2}(\mu)}{4} \bar{v}^{2}(\mu) .
$$

The pole mass of the $Z$-boson $\left(m_{Z}\right)$ is given by the $\overline{D R}$ mass and the transverse part of the $Z$-boson self-energy, so we can calculate the $\overline{D R}$ mass:

$$
\begin{aligned}
{\left.\left[p^{2}-\bar{m}_{Z}^{2}(\mu)+\operatorname{Re} \Pi_{Z}^{\mathrm{T}}\left(\mathrm{p}^{2}\right)\right]\right|_{p^{2}=m_{Z}^{2}} } & =0, \\
\bar{m}_{Z}^{2}(\mu) & =m_{Z}^{2}\left(1+\delta_{Z}(\mu)\right), \\
\delta_{Z}(\mu) & \equiv \frac{\operatorname{Re} \Pi_{Z}^{\mathrm{T}}\left(m_{Z}^{2}\right)}{m_{Z}^{2}} .
\end{aligned}
$$

In the MSSM, one loop contributions to $\delta_{Z}$ are given by, for example, Eq. (D.4) in Ref. [12]. For example, taking $m_{Z}=91.1876 \mathrm{GeV}$ and $m_{h}=115 \mathrm{GeV}$ and assuming all SUSY particle masses to be $M_{S U S Y}=1 \mathrm{TeV}$ (neglecting all mass mixings), we get

$$
\bar{v}\left(m_{Z}\right)=249.5 \mathrm{GeV} .
$$

We note that the SUSY scale $\left(M_{S U S Y}\right)$ dependence of $\bar{v}$ is very mild because the SUSY corrections to the $\overline{D R} Z$-mass are partially canceled by those from the gauge couplings. For $M_{S U S Y}=200 \mathrm{GeV}-3 \mathrm{TeV}$ and $m_{h}=115 \mathrm{GeV}$,

$$
\bar{v}\left(m_{Z}\right)=249.5 \pm 0.2 \mathrm{GeV}=249.5(1 \pm 0.08 \%) \mathrm{GeV} .
$$

The Higgs mass dependence is also very small: $\bar{v}\left(m_{Z}\right)=249.5 \pm 0.1 \mathrm{GeV}$ for $m_{h}=100-135$ GeV. Therefore, in section 3, we fix $M_{S U S Y}=1 \mathrm{TeV}$ and $m_{h}=115 \mathrm{GeV}$ for the $\bar{v}$.

Then the $\overline{D R}$ Yukawa couplings in the MSSM are given by

$$
\begin{aligned}
& \bar{y}_{t}\left(m_{Z}\right)=\frac{\sqrt{2} \bar{m}_{t}^{M S S M}\left(m_{Z}\right)}{\bar{v}\left(m_{Z}\right) \sin \beta}=\frac{\sqrt{2} \bar{m}_{t}^{S M}\left(m_{Z}\right)}{\bar{v}\left(m_{Z}\right) \sin \beta}\left(1+\delta_{t}\left(m_{Z}\right)\right), \\
& \bar{y}_{b}\left(m_{Z}\right)=\frac{\sqrt{2} \bar{m}_{b}^{M S S M}\left(m_{Z}\right)}{\bar{v}\left(m_{Z}\right) \cos \beta}=\frac{\sqrt{2} \bar{m}_{b}^{S M}\left(m_{Z}\right)}{\bar{v}\left(m_{Z}\right) \cos \beta}\left(1+\delta_{b}\left(m_{Z}\right)\right), \\
& \bar{y}_{\tau}\left(m_{Z}\right)=\frac{\sqrt{2} \bar{m}_{\tau}^{M S S M}\left(m_{Z}\right)}{\bar{v}\left(m_{Z}\right) \cos \beta}=\frac{\sqrt{2} \bar{m}_{\tau}^{S M}\left(m_{Z}\right)}{\bar{v}\left(m_{Z}\right) \cos \beta}\left(1+\delta_{\tau}\left(m_{Z}\right)\right) .
\end{aligned}
$$


where $\delta_{f}\left(m_{Z}\right)$ are the weak-scale corrections due to SUSY particle loops. A relation between the fermion pole $\left(m_{f}\right)$ and $\overline{D R}\left(\bar{m}_{f}(\mu)\right)$ masses is defined by

$$
\begin{aligned}
{\left.\left[\not p-\bar{m}_{f}(\mu)+\Sigma(p)\right]\right|_{p=m_{f}} } & =0 \\
\bar{m}_{t}(\mu) & =m_{f}\left\{1+\frac{\Sigma_{f}\left(m_{f}\right)}{m_{f}}\right\} .
\end{aligned}
$$

where $\Sigma_{f}$ is the self-energy of the fermion propagator. Therefore the SUSY contributions $\delta_{f}\left(m_{Z}\right)(f=t, b$ and $\tau)$ are defined by

$$
\delta_{f}\left(m_{Z}\right)=\frac{\Sigma\left(m_{f}\right)^{M S S M}-\Sigma\left(m_{f}\right)^{S M}}{m_{f}} .
$$

The one loop SM and SUSY contributions to $\delta_{t, b, \tau}$ can be found, for example, in Eq. (D.18) in Ref. [12].

After we get all $\overline{D R}$ gauge and Yukawa couplings at $m_{Z}$ in the MSSM, we numerically solve two loop RG equations for the full MSSM from $m_{Z}$ scale to the GUT scale in order to analyze the couplings at the GUT scale, and test for unification.

\section{References}

[1] T. Banks, "Supersymmetry And The Quark Mass Matrix," Nucl. Phys. B 303, 172 (1988); M. Olechowski and S. Pokorski, "Hierarchy Of Quark Masses In The Isotopic Doublets In N=1 Supergravity Models," Phys. Lett. B 214, 393 (1988); S. Pokorski, Nucl. Phys. (Proc. Suppl.) B13, 606 (1990); B. Ananthanarayan, G. Lazarides and Q. Shafi, "Top Mass Prediction From Supersymmetric Guts," Phys. Rev. D 44, 1613 (1991) S. Dimopoulos, L. J. Hall and S. Raby, "A Predictive framework for fermion masses in supersymmetric theories," Phys. Rev. Lett. 68, 1984 (1992); "A Predictive ansatz for fermion mass matrices in supersymmetric grand unified theories," Phys. Rev. D 45, 4192 (1992); G. W. Anderson, S. Raby, S. Dimopoulos and L. J. Hall, "Precise predictions for $m_{t}, V_{c b}$, and $\tan \beta$," Phys. Rev. D 47, 3702 (1993) hep-ph/9209250; G. Anderson, S. Raby, S. Dimopoulos, L. J. Hall and G. D. Starkman, "A Systematic SO(10) operator analysis for fermion masses," Phys. Rev. D 49, 3660 (1994) hep-ph/9308333;

[2] For example, see H. Murayama, M. Olechowski and S. Pokorski, "Viable $t-b-\tau$ Yukawa Unification in SUSY SO(10)," Phys. Lett. B 371, 57 (1996) hep-ph/9510327 and references therein.

[3] T. Blažek and S. Raby, "b $\rightarrow$ s gamma with large tan(beta) in MSSM analysis constrained by a realistic SO(10) model," Phys. Rev. D 59, 095002 (1999) hep-ph/9712257. 
[4] H. Baer et al., "Yukawa unified supersymmetric SO(10) model: Cosmology, rare decays and collider searches," Phys. Rev. D 63, 015007 (2001) hep-ph/0005027.

[5] T. Blažek, R. Dermíšek and S. Raby, "Predictions for Higgs and SUSY spectra from SO(10) Yukawa unification with mu > 0," Phys. Rev. Lett. 88, 111804 (2002) hep-ph/0107097; "Yukawa unification in SO(10)," Phys. Rev. D 65, 115004 (2002) hep-ph/0201081; R. Dermíšek "Yukawa coupling unification in SO(10) supersymmetric grand unified theories," hep-ph/0108249; S. Raby, "Testing theories of fermion masses," hep-ph/0110203;

[6] H. Baer and J. Ferrandis, "Supersymmetric SO(10) GUT models with Yukawa unification and a positive mu term," Phys. Rev. Lett. 87, 211803 (2001) hep-ph/0106352.

[7] For bottom-tau unification and other types of Yukawa unifications, see also for very recent studies, S. Komine and M. Yamaguchi, "Bottom-tau unification in SUSY SU(5) GUT and constraints from b $\rightarrow$ s gamma and muon g-2," Phys. Rev. D 65, 075013 (2002) hep-ph/0110032; U. Chattopadhyay and P. Nath, " $b-\tau$ unification, $g_{\mu}-2$, the $b \rightarrow s \gamma$ constraint and nonuniversalities," Phys. Rev. D 65, 075009 (2002) hep-ph/0110341; M. E. Gomez, G. Lazarides and C. Pallis, "Yukawa quasi-unification," Nucl. Phys. B 638, 165 (2002) hep-ph/0203131; B. Bajc, G. Senjanovic and F. Vissani, " $b-\tau$ unification and large atmospheric mixing: A case for non-canonical see-saw," hep-ph/0210207]; J. Ferrandis, "Mass predictions based on a supersymmetric SU(5) fixed point," |hep-ph/0211370.

[8] R. Hempfling, "Yukawa coupling unification with supersymmetric threshold corrections," Phys. Rev. D 49, 6168 (1994).

[9] L. J. Hall, R. Rattazzi and U. Sarid, "The Top quark mass in supersymmetric SO(10) unification," Phys. Rev. D 50, 7048 (1994) hep-ph/9306309.

[10] M. Carena, M. Olechowski, S. Pokorski and C. E. Wagner, "Electroweak symmetry breaking and bottom - top Yukawa unification," Nucl. Phys. B 426, 269 (1994) hep-ph/9402253.

[11] R. Rattazzi and U. Sarid, "The Unified minimal supersymmetric model with large Yukawa couplings," Phys. Rev. D 53, 1553 (1996) hep-ph/9505428.

[12] D. M. Pierce, J. A. Bagger, K. T. Matchev and R. j. Zhang, "Precision corrections in the minimal supersymmetric standard model," Nucl. Phys. B 491, 3 (1997) hep-ph/9606211.

[13] A. L. Kagan and M. Neubert, "QCD anatomy of B $\rightarrow$ X/s gamma decays," Eur. Phys. J. C 7, 5 (1999) hep-ph/9805303.

[14] A. Ali, E. Lunghi, C. Greub and G. Hiller, "Improved model-independent analysis of semileptonic and radiative rare B decays," Phys. Rev. D 66, 034002 (2002) hep-ph/0112300. 
[15] L. Randall and R. Sundrum, "Out of this world supersymmetry breaking," Nucl. Phys. B 557, 79 (1999) hep-th/9810155.

[16] G. F. Giudice, M. A. Luty, H. Murayama and R. Rattazzi, "Gaugino mass without singlets," JHEP 9812, 027 (1998) hep-ph/9810442.

[17] For recent discussions on this topic, see J. F. Gunion and S. Mrenna, "A study of SUSY signatures at the Tevatron in models with near mass degeneracy of the lightest chargino and neutralino," Phys. Rev. D 62, 015002 (2000) hep-ph/9906270; J. F. Gunion and S. Mrenna, "Probing models with near degeneracy of the chargino and LSP at a linear $e^{+} e^{-}$collider," Phys. Rev. D 64, 075002 (2001) hep-ph/0103167.

[18] S. P. Martin and J. D. Wells, "Super-conservative interpretation of muon g-2 results applied to supersymmetry," hep-ph/0209309.

[19] M. Davier, S. Eidelman, A. Hocker and Z. Zhang, "Confronting spectral functions from $e^{+} e^{-}$annihilation and tau decays: Consequences for the muon magnetic moment," hep-ph/0208177.

[20] T. Gherghetta, G. F. Giudice and J. D. Wells, "Phenomenological consequences of supersymmetry with anomaly-induced masses," Nucl. Phys. B 559, 27 (1999) hep-ph/9904378.

[21] T. Moroi and L. Randall, "Wino cold dark matter from anomaly-mediated SUSY breaking," Nucl. Phys. B 570, 455 (2000) hep-ph/9906527.

[22] D. Abbaneo et al. [LEP Collaboration], "A combination of preliminary electroweak measurements and constraints on the standard model," hep-ex/0112021 v2.

[23] S. Fanchiotti, B. A. Kniehl and A. Sirlin, "Incorporation of QCD effects in basic corrections of the electroweak theory," Phys. Rev. D 48, 307 (1993) hep-ph/9212285; G. Degrassi, P. Gambino and A. Sirlin, "Precise calculation of $M_{W}, \sin ^{2} \theta_{W}\left(m_{Z}\right)$, and $\sin ^{2} \theta_{\text {lept }}^{\text {eff }}$ " Phys. Lett. B 394, 188 (1997) hep-ph/9611363.

[24] K. Hagiwara et al. [Particle Data Group Collaboration], "Review Of Particle Physics," Phys. Rev. D 66, 010001 (2002). 